\title{
Face Recognition Techniques for Authentication in Smart Devices - Comparative Study
}

\author{
Jerin George \\ Post Graduate Student, MCA \\ Department of Computer Science, Christ University \\ Bengaluru, India
}

\author{
Tulasi B. \\ Department of Computer Science \\ Christ University \\ Bengaluru, India
}

\begin{abstract}
With rapid development of technology there has been a surge in hand-held devices. These devices are looked upon as an alternative to the traditional devices like personal computer and laptop. As the storage and the processing capabilities of these devices are increasing they are been termed as smart devices. The amount of personal data stored in these devices has increased many folds. In order to ensure that the critical data that is stored in these devices is secured, it is essential to put in place authentication processes. Authentication can be done at multiple levels, ranging from a password to face recognition. Algorithms like Eigenfaces, Fisherfaces are being used as a part of authentication applications. This paper tries to provide a comparative study on most commonly used face recognition algorithms.
\end{abstract}

\section{General Terms}

Face Recognition, Smart Devices, Eigenfaces Algorithm, Fisherfaces Algorithm.

\section{Keywords}

Correlation, Eigenfaces, Face Recognition, Fisherfaces, Lambertian Surface, MATLAB, Principal Components Analysis, Smart Devices.

\section{INTRODUCTION}

In the world of smart homes, smart phones, smartwatches and smart devices in general where people not just use smart devices for the basic operations such as calculations of minute numbers to finding their way back to home from work. Each and every operation is done with the help of smart technology. So, in other words smart devices have to learn their user's habits and behavior to act smart and give an appropriate response to their users.

One of the main aspects which concerns a lot is security in these devices. While using smart devices most users expect the device to recognize its user and start working. In other words, the device belonging to a certain user should not be accessible by any other unauthorized user. So to make this possible multiple security measures were created. Where the system itself can recognize its user and allow him or her to use the device. In other words the system should identify and verify its user and based on the privilege given set of action should be followed.

These recognition and identification of user can be done in many ways some of them are by comparing biometric such as fingerprint or eye iris recognition [10]. The most common one is by comparing face or in other words by face recognition. Face Recognition in short means to identify one's face as a whole by comparing a person's face with the stored data of other people's face. In the past many years, studies have been done on multiple algorithms or techniques related to face recognition. These algorithms are developed on such a way that they can give an efficient system with very less error rate.

There are many algorithms which uses different methodology [9] for face recognition. Methodologies such as facial recognition using fiducial points, facial recognition using class specific linear projection, facial recognition using multilayer perceptron, facial recognition using local feature analysis and so on are generally used in facial recognition algorithms [6, 15]. Facial recognition algorithms can be of different types $[4,16]$. In this paper, four such algorithms are examined which uses different methods to recognize a face. They are:

- Correlation - uses nearest neighbor classifier in the image space.

- Eigenfaces - uses Principal Components Analysis (PCA) to maximize the scatter of all projected samples.

- Linear Subspace - recognizes that a face is a Lambertian surface.

- Fisherfaces - uses linear subspace to find the proper match.

When looked into, studies found the best one is always the one which can recognize a person with minimal number of comparison and in very fast speed or less time. In order to identify the best method two parameters which can be considered are number of comparisons and the time taken to recognize. Using these parameters one of the method which gave a better solution is recognition based on face as a whole. And it is one of the earliest form of recognition used in almost any kind of device.

\section{SMART DEVICES}

Smart devices are devices which can partially "think"/intellect and do actions based on it. The devices which can fetch, analyze and process data by its own are generally known as smart devices. In the present fast growing society the need for smart devices are growing swiftly because of which growth in technologies related to smart devices are raising.

Lots of new technologies and devices are there through which the day to day life becomes more smoother and faster. Smart devices are now a days a big part of each and everyone's life. They are being used for each and every tasks. From the latest smart phone to innovative smart shoes and smart homes [11], the presence of smart devices is everywhere. Assisting and helping people with their daily chorus.

Smart devices have a number of advantages because of which day to day life can be more smoother. Few of the advantages are: 
- Effective Time Utilization: Because of smart devices complex operations or tasks can be done in less time when compared with doing those tasks manually. By doing so time can be saved and utilize it in somewhere else better.

- Additional Processing Power: With the help of smart devices certain simple or complex tasks (based on the level of advancement) can be given to smart devices and without much of explanation and detailing these devices do the task.

- Personal Assistance: Due to recent advancement of smart devices technology in medical field, now even if any human error happens these devices detect those errors by themselves and alert or assist the assigned personals. In other words, with the help of advancement in smart device's technology, these device can now assist humans with ease.

In the present world the use of smart devices or Internet of Things (IOT) [13] has increased drastically. Because of which all the day to day operations people generally do have shifted to devices such as smart phones and smart devices. The growth of smart devices in both technological and production perceptive has gone really high. Due to which each house has at least two or more smart devices with them.

As the smart technology got much more economical than the technology prior to them, the availability of smart devices has increased multi-folds. Due to which the usability of smart devices has increased a lot. In other terms, smart devices are being used for a small scale industry to a large scale industry.

Because of the wide range of availability and usability of smart devices, a great concern about its security arise. Rather than concerns about the physical body of the device, concern about the data generated by these devices were focused. When dealing with the data, vulnerable for the organization or for a person. Safety and security of these data from external threats are always a big concern. So to make these devices more secure and threat free lot and lots of new methods are applied. Methods such as using biometrics, passwords, and so on. Out of all these most people prefer biometrics and methods which use unique personal digital signatures such as using fingers for finger prints, iris for iris scan and face as a whole in face recognition. So using all these methods security of these devices or in other terms data generated through these devices are made more secure.

\section{FACE RECOGNITION}

The paper will be focusing on four face recognition algorithms. Each algorithm has its own method or way of analyzing and recognizing the face [18]. Generally, face recognition consists of detection and recognition. Detection takes place when the system tries to find out a face in the inputted data [2]. And recognition happens when the system tries to match up pre stored faces with the detected face [10]. So to recognize a face both the process needs to be done.

Almost all the face recognition algorithm use the above set of processes. Here the paper will be talking about four such algorithms which follows the above processes to complete their tasks. Now each of the algorithm will be explained in brief.

\subsection{Correlation}

Correlation is one of the simplest form of classification scheme in which nearest neighbor classifier in the image space is taken. In this method an image which is present in the test set or the group of images which are given for the recognition is identified and is assigned with a label of the closest point in the learning set or the group of images which are already in the dataset. In this process the distances are measured based on the image space. Once all the images in the test set and the learning set are being normalized to have a zero mean and unit variance. Then this process is similar to selecting an image from the learning set which is similar to or which correlates with the image from the test set.

Due to normalization each image gets escaped from the light source intensity i.e. if the image has an abnormal light source or the light falling on the image is not proper and the effect of grain control from a video camera i.e. the distortions happened due to a camera [9]. So in other words, because of normalization the defects happened or present in an image is ignored.

The correlation procedure has various disadvantages. Disadvantages such as:

- Variation in lighting condition leads to a loosely clustered image space.

- For accuracy, a learning set is needed with a densely sampled field of all the possible lighting conditions that may occur in the test set.

- Computationally expensive. It takes time to correlate each test image with the learning set. So to reduce the computational time, new hardware were used.

- Very large amount of storage is needed to create a learning set containing multiple images of the same person.

\subsection{Eigenfaces}

As methods such as correlation are expensive and uses huge amount of space for processing, it's common to go for other options. Eigenfaces, which uses dimensionality reduction schemes method is one of the widely used face recognition algorithm. It is preferred over other algorithms because of the technique it uses for dimensionality reduction schemes method. That is eigenfaces uses principal components analysis (PCA) technique in dimensionality reduction schemes which is generally used in computer vision or in other words technique used by computer to access and use an input device through which computer can gain some visual data [7]. PCA technique uses dimensionality reducing linear projection through which it obtains maximum scatter of the test set $[5$, 12].

Apart from its benefits, Eigenfaces technique has few disadvantages. Disadvantages such as:

- When creating scatter of an image, scatter is not only maximized between classes, but also within the classes. In short, Eigenfaces technique is less accurate than correlation.

- In class scattering happens due to different variations in light. This can be reduced by removing the significant principal components. Such as the direction where there is most variance, the directions where the data is most spread out. By doing so there is a possibility of loss of information which actually helps with facial discrimination.

\subsection{Linear Subspace}

As both correlation and eigenfaces gets affected due to variations in lighting directions, they both are not effective and accurate as needed. So to implement a better method linear subspace was taken into consideration. In linear 
subspace, all images of the face lies in a 3D linear subspace of the high dimension image space, regardless of lighting conditions. In other words, linear subspace recognizes face as a lambertian surface. Based on the amount of light directed back at the camera, the light source is determined, so with the help of minimum three images, the light source of each image could be determined, linear subspace creates the image under arbitrary lighting direction by linear combination of three original images [9]. This also states linear subspace is very accurate.

Even in linear subspace few demerits were seen. Demerits such as:

- Insensitive to a wide range of lighting conditions while only requiring three leaning set images.

- Self-shadowing, secularities, and facial expressions give the face variability that the linear subspace model does not handle as well. This can be remedied by increasing the size of the learning set, which determines which parts of the face are likely to vary.

- Computationally expensive and memory intensive.

\subsection{Fisherfaces}

Fisherfaces works in a similar way as Linear Subspaces. It uses the same technique in regards to Lambertian surface as Linear Subspaces. Fisherfaces uses Fisher's Linear Discriminant, using this technique it finds features that separates two or more classes of an object. Same as Linear Subspaces, Fisherfaces creates two scattered matrices, between class scattered matrix and within class matrix [8]. In other words, Fisherfaces takes in learning set and creates two scattered matrices out of it. One between-class scattered matrix and another one within-class matrix. Fisherfaces recognizes that variations within each class lies in a linear subspace of the image space. It follows that the classes are convex and thus linearly separable. Fisherfaces perform dimensionality reduction, while retaining linear separability. Basically, the goal is to increase distance between classes and decrease distances within classes.

Fisherfaces have a few advantages over other algorithms. Advantages such as:

- Fisher Linear Discriminant achieves greater between-class scatter than Principal Component Analysis, so this simplifies classification.

- Faster than Linear Subspaces and uses less memory.

- More accurate than Correlation and Eigenfaces.

\section{EIGENFACES AND FISHERFACES}

Eigenfaces are one of the oldest and most commonly used face recognition algorithm. From a long period of time the applications with face recognition feature are generally opting for eigenfaces as their core technique for face recognition. Even after introduction of many other algorithms with much better features eigenfaces still have the upper hand in the market. This resulted in creating many more algorithms with eigenfaces as their base. As many algorithms were developed with the help of eigenfaces, many applications which are present in the market or many applications which are yet to release, they all still use eigenfaces $[9,14]$.

Fisherfaces are one of the newly introduced algorithm in the field of face recognition. It is gaining its popularity because of many advantages this algorithm has on the existing algorithms in the market. Fisherfaces uses Fisher's Linear Discriminant because of which, features of a face can be extracted using its class separating technique. Fisherfaces are comparatively lighter than all the three algorithms. Because of which it can be considered for smart devices applications as smart devices are tend to use applications with smaller size and with more functionality.

After going through all the four algorithms (Correlation, Eigenfaces, Linear Subspace and Fisherfaces), these two algorithms (Eigenfaces and Fisherfaces) seemed to be of better fit for applications used in smart devices.

\section{IMPLEMENTATION}

Experiments were conducted so to get a better acceptable results about the accuracy and efficiency of both the algorithms. By doing so working of both the algorithms were clearer for better understanding of each algorithm. The way they processed the inputted dataset under certain conditions enabled the experiment to understand both the algorithms nature of processing data and helped with making conclusions for the experiment.

The experiments were conducted on multiple datasets. Each dataset contained faces of different people with different facial expressions and with different lighting background. Learning set was populated with images taken in proper lighting and posture. Learning set contained images or faces with proper features. So the system won't miss out or not have clear features of an image or face.

The image database used for experiments were Aberdeen database [1], Japanese Female Facial Expression (JAFFE) Database [3] and Yalefaces Database [17]. Each dataset was divided based on different race and different geographical locations. Because of which it was easier to find how both the algorithms reacted to a particular dataset.
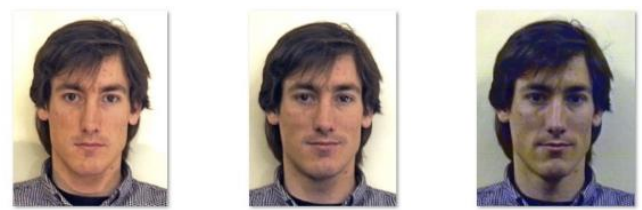

Figure 1: shows dataset with light variations. (Aberdeen Database)
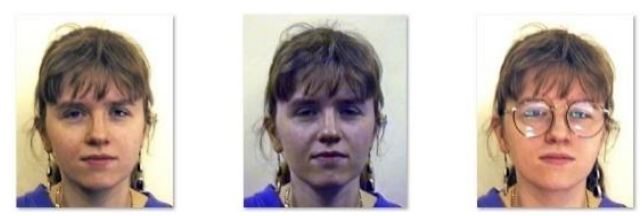

Figure 2: shows dataset with light variations and with glasses
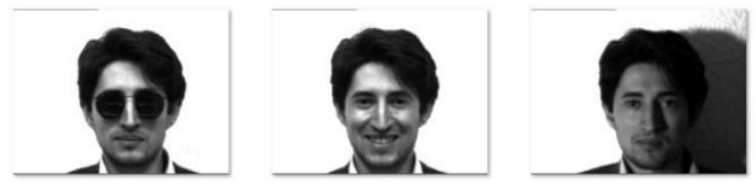

Figure 3: shows dataset with light variations, different facial expressions and with glasses 


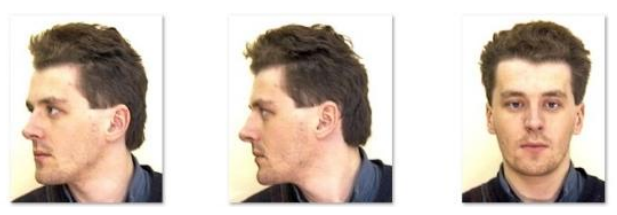

Figure 4: shows dataset containing a single face taken from different angles
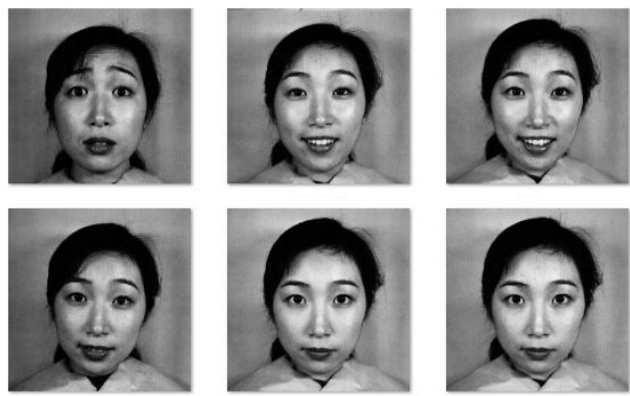

Figure 5: shows dataset with face from a different graphical location

For the experiments, MATLAB software developed by The MathWorks, Inc. was used. MATLAB is one of the most commonly used software by engineers and scientists to analyze and design systems and products. MATLAB provides a wide variety of help in the field of Math, Graphics and Programming. Using MATLAB it's easier to display results of an experiment in much more understandable graphical way.

Multiple functions were used in order to get the precise results. One such function was FaceFinder function. Where the function's basic operation was to spot the face in an image inputted to the program. In this function the face detection is happened using a specific pattern tracking method. Once FaceFinder detects a face, it then crops the image and pass it to functions named EigenFaceRunner and FisherFaceRunner. In these functions the cropped image or face is used as the input and then put into test set and is compared or recognized with the pre-loaded learning set. Once the comparison and recognition is done the rate of recognition is given. Based on this rate the end result was analyzed.

Experiments were done in two sections. First, on basis of variation in lighting and secondly, on basis of variation in lighting and facial expression together were done. In case of first section, the dataset contained images with same facial expression as of learning set but with variations in lightings. Where as in second section, images had both different facial expression and variations in lightings. So for experiments the attributes taken into consideration were number of images processed and the recognition rate.

By doing the experiments with different sections, different datasets and different technological constraints it showed that Fisherfaces has a better rate of recognition than Eigenfaces when number of images processed and the recognition rate are taken into consideration.

\section{INFERENCES}

The experiment was conducted in order to find out the best face recognition algorithm out of the existing algorithms used in the smart devices. After thorough study the list of four algorithms (Correlation, Eigenfaces, Linear Subspace and Fisherfaces) were reduced to two algorithms (Eigenfaces and
Fisherfaces) which could be easy fit for applications to be used in smart devices.

During experiment both the algorithms, Eigenfaces and Fisherfaces were given the same dataset and learning set. The output of this experiment was displayed on a graph. Output of both the algorithms were combined together and showed in one single graph rather than two different graphs. This was done so to have a better view and understanding of both the algorithms outputs. And once a common graph for both the algorithms is available, it gets easy to select the algorithm with the better output.

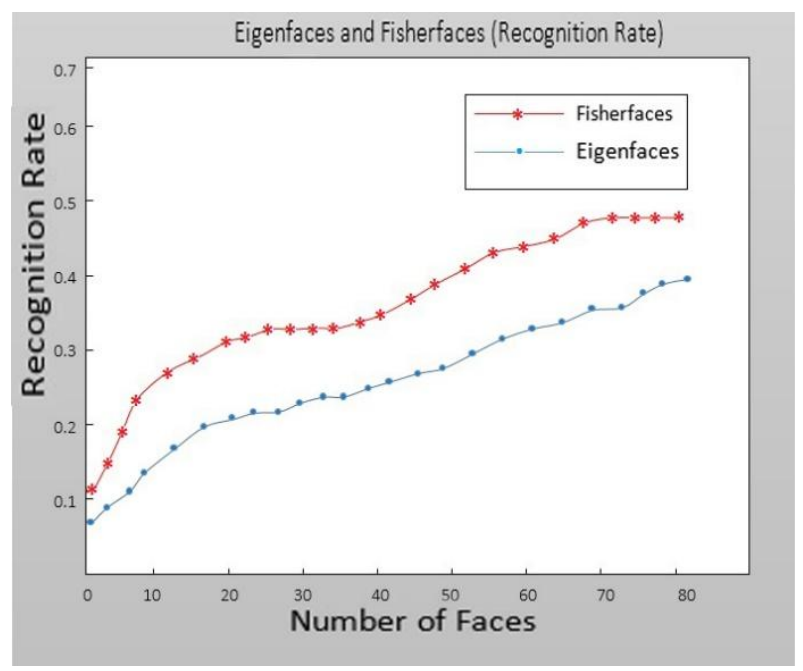

Figure 6: shows EIGENFACES and FISHERFACES recognition rate

In Figure 6, shows the combined result of Eigenfaces and Fisherfaces when given with 80 faces of dataset. With the help of this figure, the recognition rate by both the algorithms can be easily analyzed. Eigenfaces rate of recognition came out low. Whereas for Fisherfaces the initial rate of recognition was high and then after a certain amount of processing it maintained a steady rate. In other words, Eigenfaces has a lower recognition rate when compared with Fisherfaces. So with the help of experiments a conclusion can be drawn that Fisherfaces are much better than Eigenfaces.

\section{CONCLUSION}

The study has been done to find out which face recognition algorithm can be applied to the smart devices, to improve their privacy and authentication feature. The two algorithms, considered were Eigenfaces and Fisherfaces. Experiments were done on both the algorithms. The parameters considered were number of images processed and the recognition rate. The results showed that Fisherfaces was much better than Eigenfaces when given with a limited dataset. The recognition rate of Fisherfaces was much higher than Eigenfaces. Hence for an elevated level of privacy using face recognition in smart devices, and furthermore applying Fisherfaces algorithm can be considered.

\section{REFERENCES}

[1] Aberdeen Database, Web address: http://pics.stir.ac.uk/2D_face_sets.htm/, November 2016.

[2] G. Hemalatha and C. P. Sumathi, "A Study of Techniques for Facial Detection and Expression 
Classification", International Journal of Computer Science and Engineering Survey (IJCSES), Volume 5, No. 2, April 2014.

[3] Japanese Female Facial Expression (JAFFE) Database, Web address: http://www.kasrl.org/jaffe.html/, November 2016.

[4] Jigar M. Pandya, DevangRathod and Jigna J. Jadav, “A Survey of Face Recognition approach", International Journal of Engineering Research and Applications (IJERA), Volume 3, Issue 1, January - February 2013.

[5] Mandeep Kaur, Rajeev Vashisht and NirvairNeeru, "Recognition of Facial Expressions with Principal Component Analysis and Singular Value Decomposition", International Journal of Computer Applications, Volume 9, No. 12, November 2010.

[6] MarijetaSlavkovic and DubravkaJevtic, "Face Recognition Using Eigenface Approach", Serbian Journal of Electric Engineering, Vol. 9, No.1, February 2012.

[7] Matthew Turk and Alex Pentland, "Eigenfaces for Recognition", Journal of Cognitive Neuroscience, Vol. 3, No. 1, 1991.

[8] NaotoshiSeo, "Eigenfaces and Fisherfaces, 2006.

[9] Peter N. Belhumeur, Joao P. Hespanha and David J. Kriegman, "Eigenfaces vs. Fisherfaces: Recognition Using Class Specific Linear Projection", IEEE Trans. on PAMI, July 1997.

[10] RajibSaha and DebotoshBhattacharjee, "Memory Efficient Human Face Recognition Using Fiducial Points", International Journal of Advanced Research in Computer Science and Software Engineering, Volume2, Issue 1, January 2012.
[11] Satish Palaniappan, Naveen Hariharan, Naren T. Kesh, Vidhyalakshimi and Angel Deborah S., "Home Automation Systems- A study", 2015.

[12] Shiwani, Dr. Kamal Sharma and Er. Gurinder Singh, "PCA Based Improved Algorithm for Face Recognition", International Journal of Recent Research Aspects, February 2015.

[13] SomayyaMadakam, R. Ramaswamy and SiddharthTripathi, "Internet of Things [IoT]: A Literature Review", Journal of Computer and Communications, Web address: http://www.scirp.org/journal/jcc http://dx.doi.org/10.4236/jcc.2015.35021/, 2015, 3, 164173, Published Online May 2015 in SciRes.

[14] Stefano Arca, Paola Campadelli and RaffaellaLanzarotti, "A Face Recognition System Based on Local Feature Analysis", Springer-Verlag Berlin Heidelberg, 2003.

[15] Stefano Arca, Paola Campadelli and RaffaellaLanzarotti, "An Automatic Feature-Based Face Recognition System", Web address: http://www.researchgate.net/publication/4035716/, October 2003.

[16] W. Zhao, R. Chellappa, A. Rosenfeld and P. J. Phillips, "Face Recognition: A Literature Survey", ACM Computing Surveys, 2003.

[17] Yalefaces Database, Web address: http://cvc.cs.yale.edu/cvc/projects/yalefaces/yalefaces.ht $\mathrm{ml} /$, November 2016.

[18] Yi-Shin Liu, Wai-Seng Ng and Chun-Wei Liu, "A Comparison of Different Face Recognition Algorithms", 2009. 